\title{
Diseño e implementación de un sistema integrado para disminuir tiempos muertos en líneas de producción industrial.
}

\section{Design and implementation of an integrated system to reduce downtime in industrial production lines.}

\section{Recibido: 22-06-2020 /Aceptado: 20-07-2020 / Publicado: 07-08-2020}

Luis Stalin López Telenchana. ${ }^{1}$ \& José Luis Pérez Rojas. ${ }^{2}$

\begin{abstract}
.
DOI: https://doi.org/10.33262/concienciadigital.v3i3.1.1372

This research shows the implementation of an integrated system that analyzes the failure modes of the different physical assets that make up an industrial production line, in order to be able to determine in a real way the main causes and events that cause the appearance of downtime in lines. of industrial production in order to find solutions that allow reducing these times. The implementation of the system was carried out methodically in seven stages. In the first, the objective of integration is established; In the second stage, the information of the industrial process is collected and filtered in a database; the third stage generates standardization indices based on the failure modes; the fourth stage develops the exploration of informative data obtained in the collection and filtration; In the fifth stage, the appropriate mathematical model is established; the sixth stage establishes the procedure for calculating the Global Equipment Effectiveness indices; finally, the seventh stage quantitatively analyzes the results of the implementation of the integrated system. The WEKA platform was used by means of the J48 decision tree to establish a real evaluation, thus, from the implementation of the system, it was possible to achieve an increase of $4,48 \%$ in the overall efficiency of the physical assets of the production line
\end{abstract}

Keywords: Implementation, production, integrated system, dead times.

\footnotetext{
${ }^{1}$ Universidad Nacional de Chimborazo, Facultad de Ingeniería, Escuela de Ingeniería Industrial, Chimborazo Ecuador, luis.lopez@unach.edu.ec

2 Escuela Superior Politécnica de Chimborazo, Facultad de Mecánica, Chimborazo Ecuador, jose.perezl@espoch.edu.ec
} 


\begin{abstract}
Resumen
La presente investigación muestra la implementación de un sistema integrado que analiza los modos de falla de los distintos activos físicos que integran una línea de producción industrial, para poder determinar de forma real las causas principales y los eventos que provocan la aparición de tiempos muertos en líneas de producción industrial con la finalidad de encontrar soluciones que permitan disminuir dichos tiempos. La implementación del sistema se desarrolló de forma metódica en siete etapas. En la primera se establece el objetivo de la integración; en la segunda etapa se recolecta y filtra la información del proceso industrial en una base de datos; la tercera etapa genera índices de estandarización en función de los modos de falla; la cuarta etapa se desarrolla la exploración de datos informativos obtenidos en la recolección y filtración; en la quinta etapa se establece el modelo matemático adecuado; la sexta etapa establece el procedimiento para el cálculo de los índices de la Efectividad Global de Equipos; finalmente la séptima etapa analiza cuantitativamente los resultados de la implementación del sistema integrado. Se utilizó la plataforma WEKA mediante el árbol de decisión J48 para establecer una evaluación real, es así que de la implementación del sistema se pudo lograr un aumento del 4,48 \% en la eficiencia global de los activos físicos de la línea de producción.
\end{abstract}

Palabras Claves: Implementación, producción, sistema integrado, tiempos muertos.

\title{
Introducción
}

Actualmente, en el sector industrial existe la necesidad de ser cada día más competitivos, lo que obliga a las organizaciones a analizar sus procesos para obtener mayor eficiencia en las líneas de producción sin que esto signifique disminuir la calidad de los productos permitiendo de esta manera cumplir con las necesidades y expectativas de los clientes. La competitividad empresarial, en el contexto de la globalización, exige a las organizaciones para ser sostenibles en mercados nacionales e internacionales tener una administración de los procesos productivos más eficiente y eficaz de sus recursos financieros, humanos, tecnológicos, entre otros. (Labarca-Zulia et al., 2007).

Disminuir tiempos muertos a través de la detección temprana de fallos bajo normativas de modos de falla se puede llevar a efecto mediante la aplicación de modelos matemáticos cuantitativos, que como lo establece Gaither \& Frazier los modelos que evalúan la cantidad son pronósticos basados en modelos matemáticos cuya fundamentación son los datos históricos. Cabe destacar que los datos históricos desarrollados en el tiempo suelen ser importantes para el pronóstico del futuro de la producción. Todos los modelos cuantitativos con base en históricos pueden ser utilizados con series de tiempo o más conocidos como series temporales, que básicamente es un cúmulo de valores observados y determinados mediante la medición real en periodos de tiempo establecidos y progresivos en cualquier contexto operacional a nivel industrial. (Gaither \& Frazier, 2000) 
La implementación de una nueva técnica o sistema es un proceso que puede generar diferentes impactos en las líneas de producción. Estas implementaciones se las puede realizar basándose en parámetros reales de producción, por lo que la mayoría de industrias utilizan técnicas de implementación basadas en la experiencia de los actores de los procesos o con experiencias exitosas en otras empresas, pero sin la posibilidad de validar la eficiencia de las decisiones (De Toni-Meneghetti et al., 2000). La estandarización de las averías partiendo de los modos de falla nos permite crear una base de datos que distingue entre fallos funcionales y fallos técnicos indispensables para evaluar de forma real cualquier proceso productivo, y como lo señala García se define como fallo funcional al tipo de fallo que impide o interrumpe al equipo, máquina o al sistema evaluado y analizado cumplir la función requerida del activo físico. A la vez el fallo técnico se determina como aquel que no impidiendo al sistema, equipo o máquina que cumpla la función requerida, además supone que el funcionamiento será anormal por lo tanto tendremos problemas que pueden ir desde la falta de calidad en los productos desarrollados o en los servicios brindados hasta paros en los procesos productivos a nivel industrial. (García Garrido, 2003)

La explotación de datos se lo realiza a través de la técnica denominada minería de datos, los objetivos centrales de la minería de datos pueden resumirse en dos grandes grupos: Describir y Predecir, lo cual podría asemejarse a procesos de estadística descriptiva e inferencial, pero debido a que la información para la toma de decisiones que proveen los algoritmos de descripción y predicción de la minería de datos, poseen una característica de hipótesis, a saber, con respecto al número de grupos o clúster que se desean, a la variable endógena por la cual se opta o al número máximo de nodos esperado; dichos algoritmos van más allá de la estadística descriptiva clásica y proponen ciertas restricciones en los resultados entregados. (Han-Pei, et al., 2012).

Existen distinta investigaciones que relacionan la aparición de tiempos muertos en función de modos de falla, esto se debe a que el sistema diseñado busca un estudio de forma integral de la líneas de producción, partiendo del principio que según Niebel \& Freivalds en el que el gerente de control de la producción es el que tiene como principal función el establecimiento y mantenimiento de programas maestros de producción teniendo en cuenta las necesidades y exigencias del cliente y por supuestos del cambiante mercado que para un adecuado análisis se realiza mediante una programación cuidadosa, mientras que el gerente de mantenimiento debe realizar los máximos esfuerzos para minimizar los tiempos muertos que aparecen de los modos de fallas y sobre todo de las reparaciones no programadas que provocan paros imprevistos en la producción. (Niebel \& Freivalds, 2009)

Al realizar el análisis bibliográfico de los distintos modelos creados para detectar y disminuir tiempos muertos en líneas de producción, podemos determinar y expresar que existen metodologías que tratan de resolver este problema, no obstante esto no implica que una metodología no pueda seguir siendo mejorada, o que sea el punto de partida para otra metodología o sistema como las herramientas WEKA y el árbol de decisiones J48, que son utilizadas en distintas aplicaciones y estudios no pierde vigencia ya que contribuye a mejorar y eliminar los tiempos muertos, puesto que se puede establecer los fallos en un árbol de decisión 
para detallar y sintetizar los datos informativos, con la finalidad de generar un sistema integrado que sin importar la línea de producción se pueda desarrollar un plan maestro de producción, que como manifiesta Chapman se trata de un proceso que tiene como fundamento el inicio en el pronóstico bastante detallado de cada uno de los productos o servicios, posteriormente se desarrolla un conjunto específico de "reglas", cuya proyección es generar pedidos reales de los clientes "consuman" dicho pronóstico, asegurando de esta manera índices de productividad en función de los requerimientos del mercado. (Chapman, 2006).

Se describe además de forma detallada las etapas para una adecuada implementación de un sistema integrado que mejora de forma sustancial la eficiencia y eficacia de cualquier línea de producción, puesto que es un sistema versátil y que da resultados porque analiza las fallas de los activos físicos de cualquier línea de producción, utilizando técnicas inteligentes como la minería de datos con la finalidad de establecer un modelo matemático apropiado, que como establece Pérez una vez establecido y configurado dicho modelo matemático se llega a predecir y a determinar el valor cuantitativo de las variables dependientes que permite establecer el perfil de las demás variables. Cabe recalcar que si la variable dependiente es cualitativa dicotómica (1 y 0 ; sí y no) puede ser empleada como clasificadora, ya que se estudia y establece la relación con las demás de variables que son clasificadas y establecidas a través del modelo de regresión logística aplicable a sistemas productivos. (Pérez Marqués , 2014)

A través de la herramienta de minería de datos con la aplicación WEKA se pudo relacionar los diferentes modos de fallas y determinar cuáles fueron los más comunes con el objetivo de plantear soluciones que posteriormente fueron evaluadas para establecer su verdadero impacto, finalmente se estableció los índices de productividad que permitieron medir de forma cuantitativa la eficiencia de la implementación del sistema integrado desarrollado en la línea de producción intervenida, que como señala Mora los sistemas integrados son aquellos que permite determinarlos en un sistema o enfoque de ingeniería de procesos, que son mantenedores o mantenimiento, los producción y los activos físicos; es así que se puede establecer varias reglas y leyes de mantenimiento industrial, en las cuales con la ayuda del sistema kantiano podemos determinar la relación que existe entre el departamento de producción y las máquinas, que se convierten en el efecto integrador de la denominas ingeniería de fábricas o plantas industriales. (Mora Gutiérrez, 2009)

Con fundamentos en la información normada referente a modos de falla que podemos encontrar en cualquier sistema productivo y que están estrechamente ligadas a la información real de la planta de producción o de servicios se propone e implementa un modelo integral de siete etapas progresivas que busca ser estándar industrial adecuado en función de los distintos sistemas de producción, siendo el sistema aplicable sin importar el tipo de fallas encontrados en el sistema productivo, pues estos rubros entran en los denominados costos de calidad, que como lo explica Render las fallas internas son costos provocados al producir o elaborar partes, productos o servicios defectuosos antes de que los mismos sean entregados a los clientes, entre ellos tenemos trabajo repetido, desperdicio de materia prima, y los tiempos muertos en producción. (Render \& 
Heizer, 2014) La presente investigación diseña e implementa una metodología capaz de determinar de forma real las causas principales y los eventos que provocan la aparición de tiempos muertos en líneas de producción industrial con la finalidad de encontrar soluciones reales y establecer resultados basados en la información en planta mediante la construcción de un modelo matemático que desarrolla planes de acción efectivos y tomas de decisión elocuentes, que como lo indica Hamdy la construcción adecuada de un modelo matemático implica de forma tácita un intento de establecer y transformar la definición teórica de cualquier problema en distintas relaciones matemáticas. Se debe tomar en cuenta que el modelo matemático resultante parte de distintos modelos matemáticos estandarizados, tales como la denominada programación lineal, que suele determinar de manera confiable una solución utilizando los algoritmos aplicables disponibles a líneas de producción.

Cabe establecer que una simulación matemática puede establecer y combinar varios modelos matemáticos heurísticos que son empleados para resolver distintos problema en la toma adecuada de decisión a nivel industrial. (Hamdy, 2012).

\section{Metodología}

La metodología de implementación propuesta se describe varias etapas, basadas en la minería de datos, por lo que se convierte en una metodología inteligente y aplicable a cualquier línea de producción pues para del análisis de modos de falla que se dan en los distintos active que integran dicha línea, lo que garantiza un impacto real al término de la implementación, ello se puede evidenciar en la última etapa en donde la metodología realiza comparaciones cuantitativas de la eficiencia y eficacia de la línea de producción antes y después de la aplicación propuesta y desarrollada en siete etapas:

- En la primera se establece el objetivo de la investigación.

- En la segunda etapa se recolecta y filtra la información del proceso industrial en una base de datos.

- La tercera etapa genera índices de estandarización en función de los modos de falla.

- La cuarta etapa se desarrolla la exploración de datos informativos obtenidos en la recolección y filtración.

- En la quinta etapa se establece el modelo matemático adecuado.

- La sexta etapa establece el procedimiento para el cálculo de los índices de la Efectividad Global de Equipos.

- Finalmente la séptima etapa analiza cuantitativamente los resultados de la implementación del sistema integrado.

Las siete etapas del sistema integrado son progresivas, de fácil entendimiento y aplicación, y en conjunto buscan ser las soluciones eficiente y eficaz para disminuir tiempos muertos en líneas de producción mediante evaluaciones cuantitativas.

Establecer el objetivo de la integración. 
En esta primera etapa el establecimiento del objetivo de la integración consiste en definir el alcance del sistema integrado y elaborar un plan de producción que incluya un cronograma de actividades con fechas de vencimiento. Esto permite cumplir un cronograma que evite una implementación inadecuada, además que se convierte en el punto de partida para realizar cambios que permitan aumentar índices de producción sin que impliquen generar mayores gastos.

Figura 1. Cronograma de actividades.

\begin{tabular}{|c|c|c|c|c|c|c|c|c|c|}
\hline Tareas & Inicio & Fin & Días & $9 / 2 \quad 9 / 3$ & $\begin{array}{lll}3 & 9 / 4 & 9 / 5\end{array}$ & $\begin{array}{llll}5 & 9 / 6 & 9 / 7\end{array}$ & 7 9/8 & & \\
\hline Fase 1 & & & & & & & & & \\
\hline 1. Establecer el objetivo de la integración. & $5 / 2$ & $5 / 30$ & $1 / 28$ & & & & & & \\
\hline 2. Recolectar y filtrar la información de la línea de producción. & $6 / 1$ & $6 / 30$ & $1 / 29$ & & & & & & \\
\hline 3. Generar índices de estandarización en función de los modos de falla & $7 / 4$ & $7 / 28$ & $1 / 24$ & & & & & & \\
\hline Fase 2 & & & & & & & & & \\
\hline 4. Desarrollar la exploración de datos informativos. & $8 / 1$ & $8 / 29$ & $1 / 28$ & & & & & & \\
\hline 5. Establecer el modelo matemático adecuado. & $9 / 2$ & $9 / 29$ & $1 / 27$ & & & & & & \\
\hline $\begin{array}{l}\text { 6. Determinar el procedimiento para el cálculo de los índices de la } \\
\text { Efectividad Global de Equipos }\end{array}$ & 10/1 & $10 / 30$ & $1 / 29$ & & & & & & \\
\hline $\begin{array}{l}\text { 7. Analizar cuantitativamente los resultados de la implementación del } \\
\text { sistema integrado. }\end{array}$ & $11 / 9$ & $11 / 30$ & $1 / 21$ & & & & & & \\
\hline
\end{tabular}

Fuente: Los Autores 2020.

\section{Recolectar y filtrar la información.}

En la segunda etapa se recolecta y filtra la información del proceso industrial en una base de datos

En esta etapa se recolecta in situ la información de las distintas novedades desarrolladas en los activos físicos que integran la línea de producción intervenida, esta información es integrada a un referente de datos que involucran varias causas con sus modos de falla respectivos de las líneas de producción intervenidas, que son generados desde dos fuentes directas, la primera fuente técnica de las distintas bitácoras de mantenimiento y la segunda los registros de intervenciones realizadas a los activos físicos de la línea de producción evaluada.

Figura 2. Recolección de información.
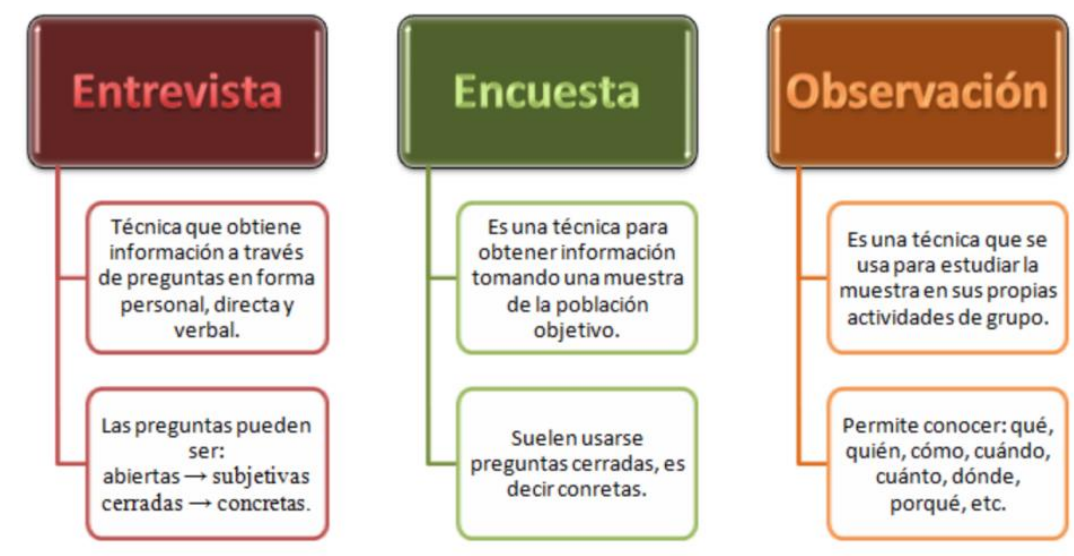

Fuente: https://normasapa.net/tecnicas-recoleccion-datos/

La segunda fuente son los datos recolectados a través de un seguimiento realizado durante el primer trimestre del año en la línea de producción a través la aplicación de formatos estándar propios de mantenimiento y producción. 


\section{Estandarizar el proceso en función de los modos de falla.}

La tercera etapa genera índices de estandarización en función de los modos de falla preestablecidos dentro del sistema productivo. Se estandariza los modos de falla empleando la Norma UNE-EN 13306 para con ello realizar un primer filtrado de los datos que nos serán útiles dentro del programa de mantenimiento, con el objetivo de relacionar, registrar y clasificar la información en función de las variables otorgados por la línea de producción, que básicamente establecen los orígenes de las causas que provocan tiempos muertos en las líneas de producción intervenidas. Una vez desarrollada la estandarización de los modos de falla, se los debe clasificar técnicamente empleando el Diagrama de Pareto, que básicamente establece que el $80 \%$ de los fallos encontrados en la línea de producción intervenida son fundamentalmente provocados por el $20 \%$ de las causas principales, de esta manera podemos establecer el deterioro y el desgaste de una línea de producción que genera tiempos muertos y problemas de producción.

Figura 1. Diagrama de Ishikawa.

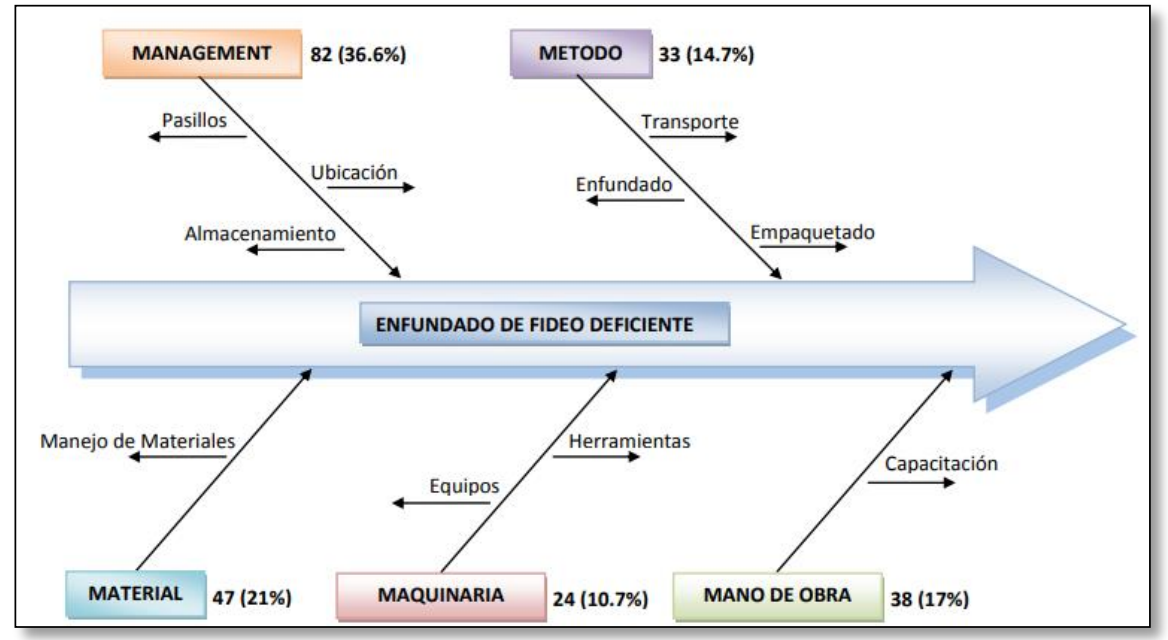

Fuente: Los Autores 2020

Figura 4. Aplicación del Diagrama de Pareto

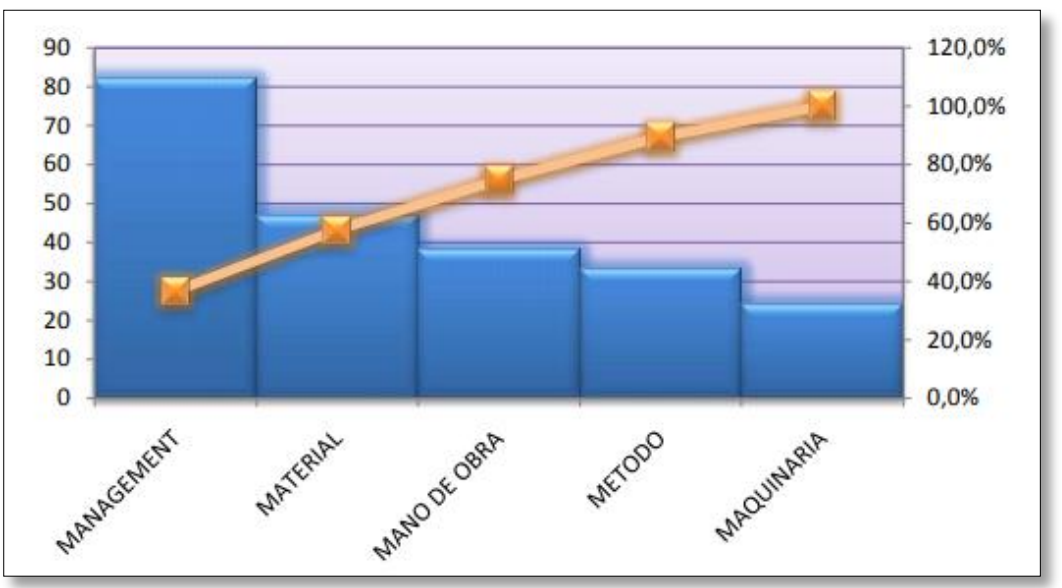

Fuente: Los Autores 2020. 


\section{Explorar los datos del proceso productivo.}

La cuarta etapa se desarrolla la exploración de datos informativos obtenidos en la recolección y filtración. La cuarta etapa emplea el programa WEKA para establecer de forma implícita las verdaderas fuentes que provocan y generan los denominados tiempos muertos, pues analiza las distintas variables de la línea de producción, empezando por realizar un diagrama de flujo acorde con la línea de producción intervenida, estableciendo la descripción integral del proceso y los tiempos adecuados y predeterminados en función de estándares de ejecución.

Figura 5. Diagrama de proceso.

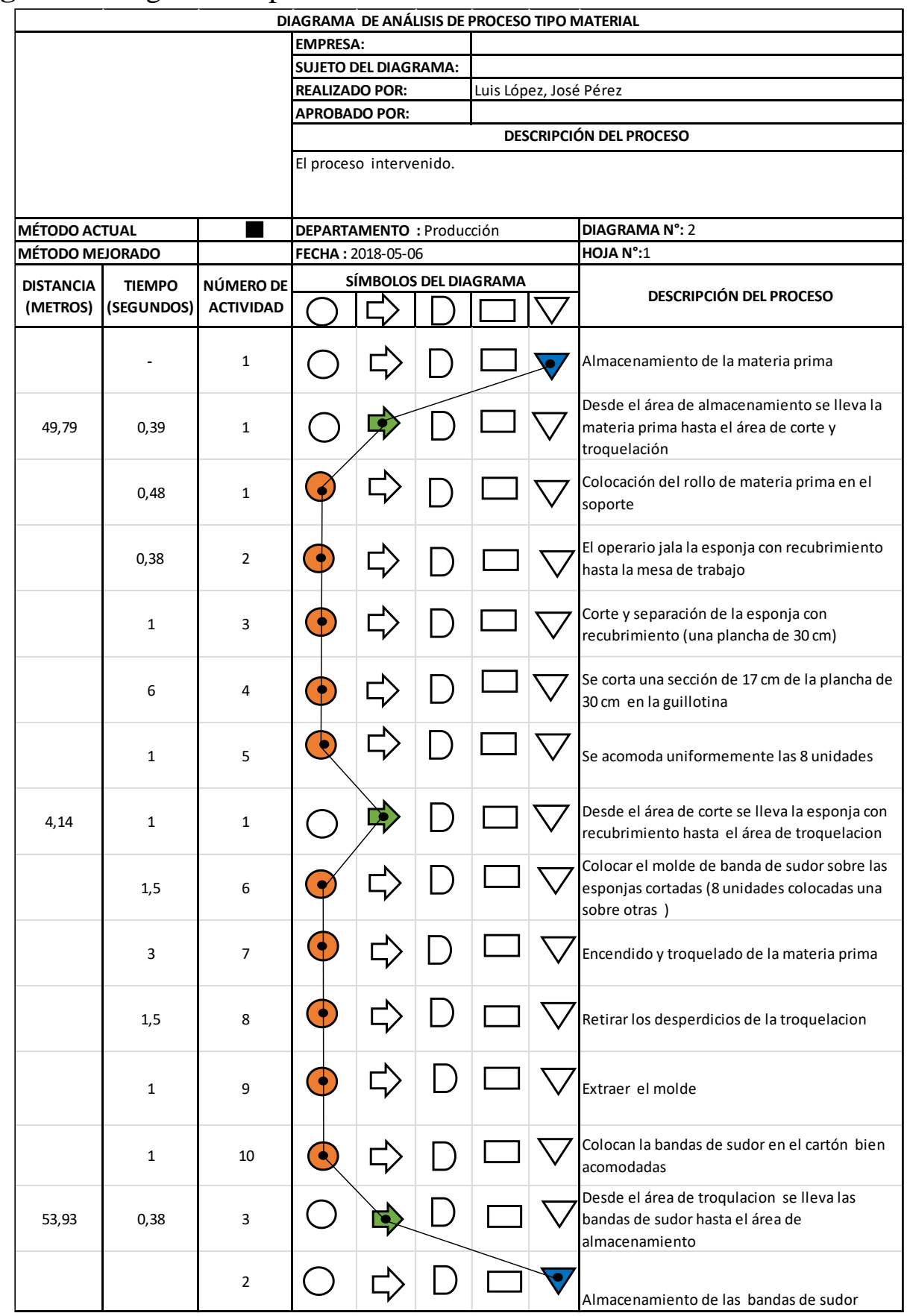

Fuente: Los Autores 2020. 
Para terminar la exploración de los datos del proceso se debe estandarizar los datos que se obtuvieron en la intervención, esto con la finalidad que en caso de que esta información sea subida a una plataforma digital no existan inconvenientes, pues se encontrará información filtrada y clasificada en niveles que permitan establecer distintos atributos referentes a la línea de producción.

\section{Aplicar un modelo matemático apropiado.}

En la quinta etapa se establece el modelo matemático adecuado. Para establecer el modelo matemático apropiado se buscó la adaptación ideal entre un modelo que permita una evaluación integral del sistema y un algoritmo que entregue resultados fiables, es así que se emplea el algoritmo J48, pues es un clasificador basado en los árboles de decisión.

Al ser un árbol de decisiones permite una coyuntura ideal entre el análisis matemático y la evaluación cuantitativa del sistema de producción, el gráfico tipo árbol de nuestro estudio se estipula a continuación, lo que permite establecer la metodología apropiada para evaluar los distintos componentes de la líneas de producción y generar una solución apropiada, hay que tener en cuenta que estas alternativas encontradas faciliten generar programas de mantenimiento adecuados que sean útiles para desarrollar planes maestros de producción. En la figura siguiente se establece los distintos sistemas y se puede identificar de forma fácil que los atributitos enmarcados en el área mecánica son los que generan más tiempos muertos en la línea de producción, partiendo de este análisis jerárquicamente hablando los atributos en función de las condiciones superan a los atributos generados en la parte eléctrica.

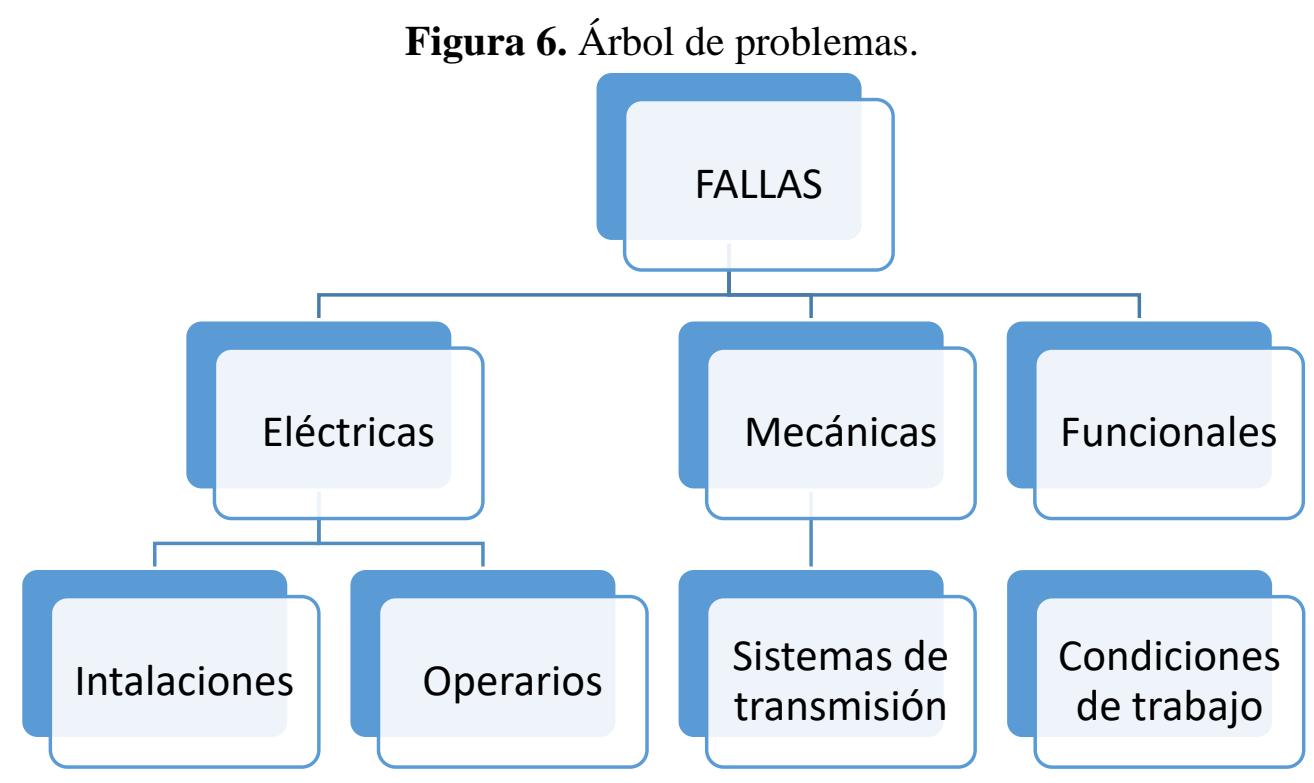

Fuente: Los Autores 2020.

El árbol presentado anteriormente se basa en el establecimiento del grupo de fallas detectado en el sistema de producción, por otro parte se debe recalcar que los activos se encuentran en 
funcionamiento y aunque trabajan en falla no han pasado a avería, por lo que los tiempos muertos han sido detectados de manera más pronto.

\section{Calcular de la Efectividad Global de Equipos.}

La sexta etapa establece el procedimiento para el cálculo de los índices de la Efectividad Global de Equipos.

OEE es básicamente una técnica que mide de forma integral cualquier línea de producción, y se ha convertido en una herramienta importante para evaluar la implementación de distintas técnica, modelos y sistemas de nivel industrial. Al hablar de distintos procesos podemos establecer una compactación de indicadores de gestión a nivel industrial como la disponibilidad de activos, eficiencia de los procesos y control de la calidad de los productos elaborados, el cálculo de este indicador global permite de forma efectiva y eficiente detectar fallos ocultos que pueden generar paros imprevistos, además de ello permite también evaluar procesos deficientes en la elaboración de productos o en la oferta de servicios. Hay que recalcar que el éxito en la ejecución y el cálculo de este importante indicador depende del nivel de compromisos de los actores involucrados en el sistema de producción, y este compromiso generará soluciones efectivas que permutan tomas de decisiones elocuentes y tangibles en el tiempo.

Se debe recordar también que la Efectividad Global de Equipos es la herramienta de nos ayuda a establecer cuando contratar más personal, cuando comprar nuevos equipos o máquinas, así también cuando adquirir materiales y repuestos, en función de una planificación, es decir es recomendable el cálculo de esta efectividad de forma anual y evaluar el sistema de la misma forma, así convirtiéndose una herramienta de calidad con al que se puede certificar en ISO 9001 cualquier sistema de producción.

La Efectividad Global de Equipos es una herramienta de calidad que combina múltiples aspectos de producción y de mantenimiento, además de establecer puntos de referencia para proporcionar viable información sobre el proceso intervenido, se la denomina herramienta integral de evaluación comparativa pues sirve para determinar los diferentes subcomponentes de una línea de producción empleando indicadores de calidad y mantenimiento, se utiliza para medir las mejoras propuestas de forma real en función de la aplicación de distintas técnicas como las 5S, Kaizen, Seis Sigma y T.P.M.

La OEE es empleada para medir y evaluar la eficiencia de cualquier proceso o línea de producción, pues se basa en tres indicadores fundamentales de gestión como disponibilidad, el rendimiento y calidad del producto, al punto de ejecutar un modelo calculado para estandarizar el proceso, buscando las causas de los tiempos muertos y establecer los distintos problemas en busca de las mejores soluciones aplicables, al ser una evolución cuantitativa podemos resumir la forma de su cálculo como lo indica la figura de abajo: 
Figura 7. Cálculo de la Efectividad Global de Equipos.

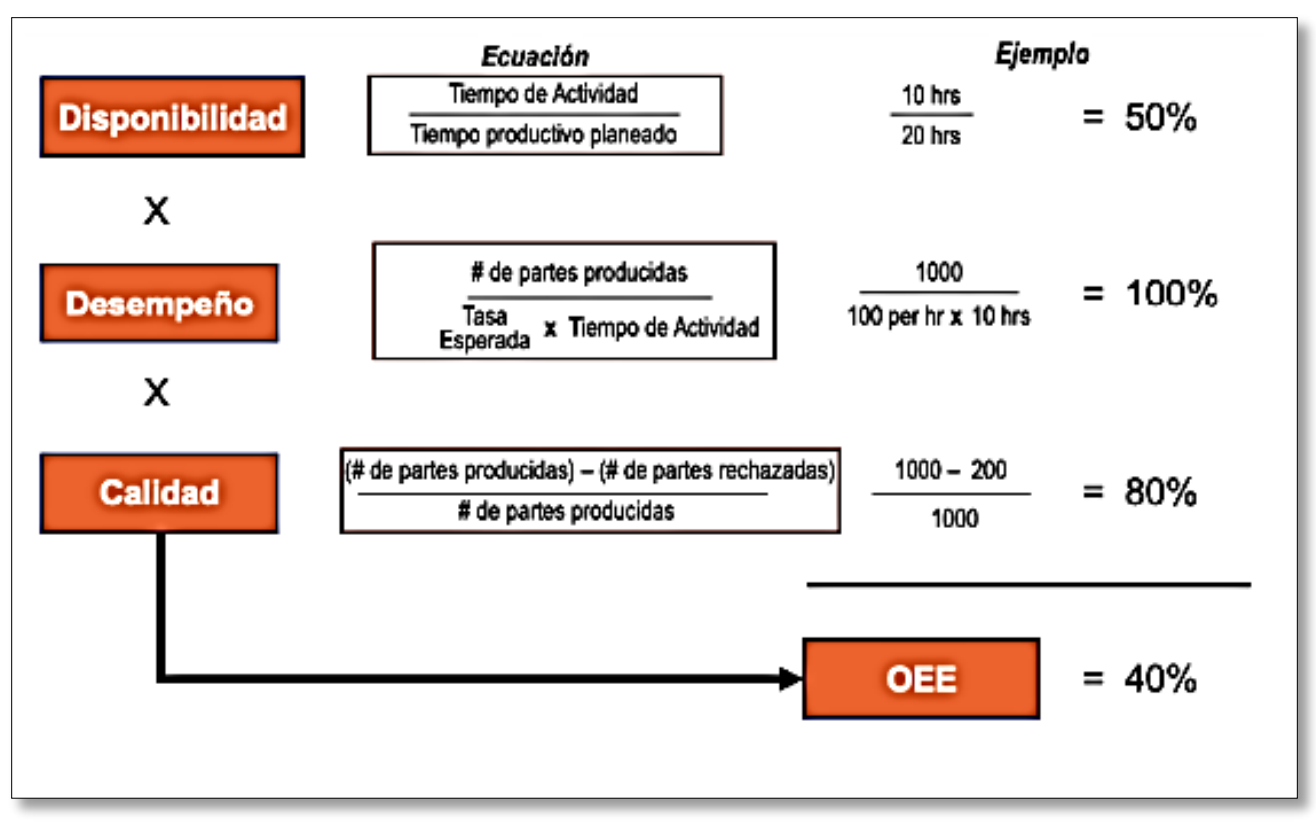

Fuente: https://www.oeecaricam.com/oee

\section{Analizar cuantitativamente los resultados.}

Finalmente la séptima etapa analiza cuantitativamente los resultados de la implementación del sistema integrado, con esta evaluación cuantitativa realizamos la respectiva comparación con los parámetros mostrados en la figura de abajo, con ello podemos describir de manera estandarizada la línea de producción intervenida.

Tabla 1. Evaluación de la Efectividad Global de Equipos.

\begin{tabular}{ccc}
\hline OEE & CALIFICACIÓN & CARACTERÍSTICAS \\
$<65 \%$ & Inaceptable & $\begin{array}{c}\text { Provoca varias pérdidas económicas. } \\
\text { La competitividad es muy baja. }\end{array}$ \\
$\geq 65 \%<75 \%$ & Regular & Sistema considerable Aceptable sólo si se implementa un \\
proceso de mejora.
\end{tabular}

Fuente: Autores 2020. 


\section{OEE INICIAL}

Para evaluar la OEE inicial se tomaron las siguientes condiciones iniciales antes de implementar el modelo integral:

Tabla 2. Condiciones Iniciales de la línea de producción.

\begin{tabular}{cc}
\hline İTEM & VALOR \\
\hline Capacidad de producción & 2000 unidades/hora. \\
Periodo de evaluación & 8760 horas. \\
TTR & 225 horas \\
Tiempo total de paros no programados & 115 horas \\
Unidades producidas & 14162440 unidades \\
Unidades defectuosas & 13592 unidades fueron defectuosas \\
Unidades no conformes de manera irreversible & 6243 unidades \\
Unidades reprocesadas & 6243 unidades \\
\hline
\end{tabular}

Fuente: Autores 2020.

Metodología:

- Tiempo Total de Producción (TPO)

TPO $=$ TTE - TTP

TPO $=$ Tiempo Total de Evaluación - Tiempo Total de Paradas Programadas

$\mathrm{TPO}=8760$ horas -225 horas

$\mathrm{TPO}=8535$ horas.

- Tiempo de Operación (TO)

$\mathrm{TO}=\mathrm{TPO}-\mathrm{TTPN}$

TO = Tiempo Total de Producción - Tiempo Total de horas por paros no programados

$\mathrm{TO}=8535$ horas -115 horas

$\mathrm{TO}=8420$ horas.

- Disponibilidad

Disponibilidad $=\mathrm{TO} / \mathrm{TPO}$

Disponibilidad $=8420$ horas $/ 8535$ horas

Disponibilidad $=0,98$

- Tiempo de Ciclo Ideal

Tiempo de Ciclo Ideal = 1/(Capacidad Nominal $)$

Tiempo de Ciclo Ideal $=1 /(2000$ unidades/hora $)$

Tiempo de Ciclo Ideal $=0,0005$ horas/unidades

- Rendimiento

Rendimiento $=$ Tiempo de Ciclo Ideal $/(\mathrm{TO} /$ Total de Unidades Producidas $)$

Rendimiento $=0,0005$ horas/unidades / ( 8420 horas / 14162440 unidades $)$

Rendimiento $=0,841$

- Calidad

Calidad $=$ Unidades Conformes $/$ Total de Unidades Producidas

Calidad $=(14162440$ unidades -19835 unidades $) / 14162440$ unidades

Calidad $=0,998$ 
Con los tres parámetros fundamentales podemos ya calcular la OEE:

- Efectividad Global de Equipos.

OEE $=$ Disponibilidad $\times$ Rendimiento $\times$ Calidad

$\mathrm{OEE}=0,98 \times 0,841 \times 0,998=0,8225$

OEE porcentual $(\%)=82,25 \%$

Se puede clasificar al sistema como ACEPTABLE.

\section{OEE FINAL}

La segunda evaluación de la OEE inicial se realizó después de implementar el modelo integral:

Tabla 3. Condiciones Finales de la línea de producción.

\begin{tabular}{cc}
\hline İTEM & VALOR \\
\hline Capacidad de producción & 2000 unidades/hora. \\
Periodo de evaluación & 8760 horas. \\
TTR & 172 horas \\
Tiempo total de paros no programados & 76 horas \\
Unidades producidas & 14894790 unidades \\
Unidades defectuosas & 9447 unidades \\
Unidades no conformes de manera irreversible & 4963 unidades \\
Unidades reprocesadas
\end{tabular}

Fuente: Autores 2020.

Metodología:

- Tiempo Total de Producción (TPO)

TPO $=$ TTE - TTP

TPO $=$ Tiempo Total de Evaluación - Tiempo Total de Paradas Programadas

$\mathrm{TPO}=8760$ horas -172 horas

$\mathrm{TPO}=8588$ horas.

- Tiempo de Operación (TO)

$\mathrm{TO}=\mathrm{TPO}-\mathrm{TTPN}$

TO $=$ Tiempo Total de Producción - Tiempo Total de horas por paros no programados

$\mathrm{TO}=8588$ horas -76 horas

$\mathrm{TO}=8512$ horas.

\section{- Disponibilidad}

Disponibilidad $=\mathrm{TO} / \mathrm{TPO}$

Disponibilidad $=8512$ horas $/ 8588$ horas

Disponibilidad $=0,99$

\section{- Tiempo de Ciclo Ideal}

Tiempo de Ciclo Ideal $=1 /($ Capacidad Nominal $)$

Tiempo de Ciclo Ideal $=1 /(2000$ unidades $/$ hora $)$

Tiempo de Ciclo Ideal $=0,0005$ horas/unidades

\section{- Rendimiento}

Rendimiento $=$ Tiempo de Ciclo Ideal $/(\mathrm{TO} /$ Total de Unidades Producidas $)$

Rendimiento $=0,0005$ horas/unidades / (8512 horas / 14894790 unidades)

Rendimiento $=0,874$ 


\section{- Calidad}

Calidad $=$ Unidades Conformes $/$ Total de Unidades Producidas

Calidad $=(14894790$ unidades -14410 unidades $) / 14894790$ unidades

Calidad $=0,999$

Con los tres parámetros fundamentales podemos ya calcular la OEE:

\section{- Efectividad Global de Equipos.}

OEE $=$ Disponibilidad $\times$ Rendimiento $\times$ Calidad

$\mathrm{OEE}=0,99 \times 0,874 \times 0,999=0,8643$

OEE porcentual $(\%)=86,43 \%$

Se puede clasificar al sistema como BUENO.

\section{Discusión}

Se puede comparar cuantitativamente el sistema de producción intervenido, antes y después de aplicar el modelo diseñado, obteniendo resultados significativos, que son traducido a una ganancia porcentual de la Efectividad Global de Equipos, consiguiendo de esta manera ser efectivos y eficaces, sin que esto signifique una mayor inversión, obteniendo resultados alentadores en al ámbito de la producción, como se muestra a continuación:

OEE INICIAL DEL PROCESO $(\%)=82,25 \%$

Se puede clasificar el OEE inicial del proceso como ACEPTABLE.

OEE FINAL DEL PROCESO $(\%)=86,43 \%$

Se puede clasificar el OEE inicial del proceso como BUENO.

El aumento porcentual que se pudo conseguir en la línea de producción es de 4,18 \%, pasando nuestro proceso intervenido de Aceptable a un estado de Bueno, consiguiendo así un incremento visible en la productividad.

\section{Conclusiones}

- Según los resultados arrojados en la presente investigación se concluye que al aplicar el sistema integrador se logra aumentar la Efectividad Global de Equipos que integran el proceso productivo haciéndolo más eficiente en función de los modos de falla analizados en un periodo de tiempo determinado.

- Se establece que para una aplicación adecuada de algoritmos en la explotación de datos el programa WEKA brinda las condiciones adecuadas y permite identificar de manera fácil los modos de falla que pasan hacer averías, las mismas que causan tiempos muertos en la línea de producción intervenida.

- Para establecer el modelo matemático apropiado se diseña la adaptación ideal entre un modelo que permita una evaluación integral del sistema y un algoritmo que entregue resultados fiables, es así que se emplea el algoritmo J48, pues es un clasificador basado en los árboles de decisión, permitiendo una evaluación real del sistema de producción. 
- Se determina que un sistema integrado evaluado mediante la identificación de las causas de los modos de falla, a su detección temprana y a su pronta resolución, permitió establecer un aumento porcentual en la línea de producción de 4,18\%, pasando nuestro proceso intervenido de Aceptable a un estado de Bueno, de esta manera podemos aseverar que el sistema integrado dio los resultados esperados haciendo más rentable el proceso.

\section{Referencias Bibliográficas}

Labarca, N. and Zulia, U. (2007.) "Consideraciones teóricas de la competitividad empresarial”, Omnia, Vol. 13, No. 2, pp. 158-184.

De Toni, A. and Meneghetti, A. (2000). "The production planning process for a network of rms in the textile apparel industry," Int. J. Prod, Vol. 65, 2.

HAN, J. y KAMBER, M. 2012. Data Mining, Concepts and Techniques. Morgan Kaufmann Publishers.

Chapman, S. N. (2006). Planificación y control de la producción. Naucalpan de Juárez, Edo. de México: Pearson Educación.

Gaither, N., \& Frazier, G. (2000). Administración de la producción y operaciones. México D.F.: International Thomson.

García Garrido, S. (2003). Organización y gestión integral de mantenimiento. Madrid: Ediciones Díaz de Santos, S. A.

Hamdy, T. (2012). Investigación de operaciones. Naucalpan de Juárez, Estado de México: PEARSON EDUCACIÓN.

Mora Gutiérrez, L. A. (2009). Mantenimiento. Planeación, ejecución y control. México D.F.: Alfaomega Grupo Editor, S.A.

Niebel, B. W., \& Freivalds, A. (2009). Ingeniería industrial: Métodos, estándaresy diseño del trabajo. México, D. F.: McGRAW-HILL/Interamericana Editores, S.A.

Pérez Marqués , M. (2014). Minería de Datos a través de ejemplos. Madrid: RC Libros.

Render, B., \& Heizer, J. (2014). Principios de administración de operaciones. Naucalpan de Juárez, Estado de México: Pearson Educación.

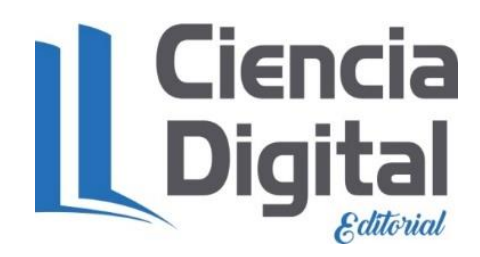




\section{PARA CITAR EL ARTÍCULO INDEXADO.}

López Telenchana, L. S., \& Pérez Rojas, J. L. (2020). Diseño e implementación de un sistema integrado para disminuir tiempos muertos en líneas de producción industrial. ConcienciaDigital, 3(3.1), 126-141. https://doi.org/10.33262/concienciadigital.v3i3.1.1372

\section{Ciencia \\ LDigital}

El artículo que se publica es de exclusiva responsabilidad de los autores y no necesariamente reflejan el pensamiento de la Revista Conciencia Digital.

El artículo queda en propiedad de la revista y, por tanto, su publicación parcial y/o total en otro medio tiene que ser autorizado por el director de la Revista Conciencia Digital.

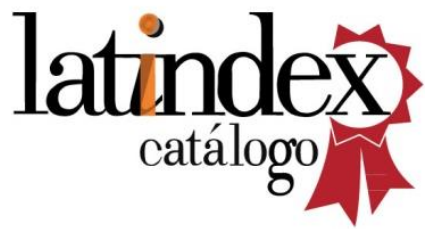

